Biotechnology

for Biofuels

D-Galacturonic acid
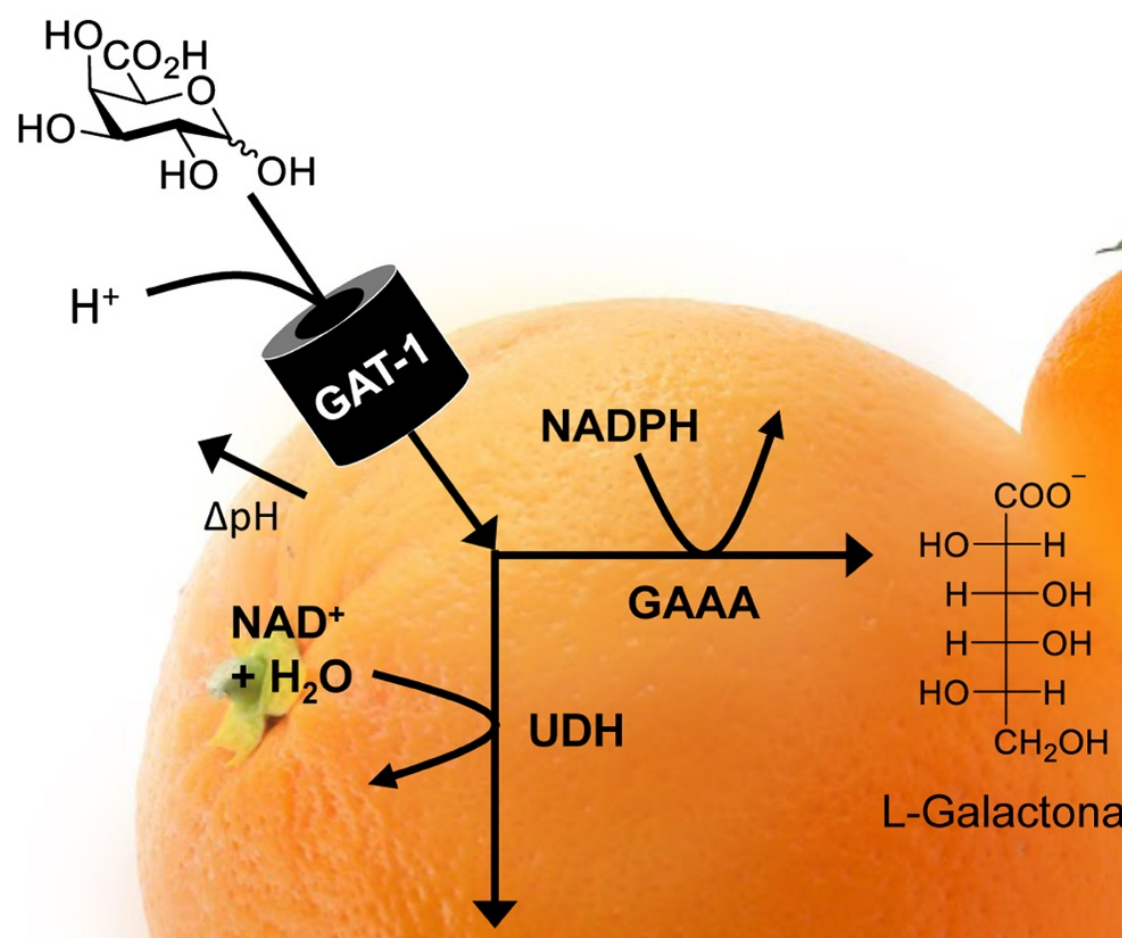

L-Galactonate

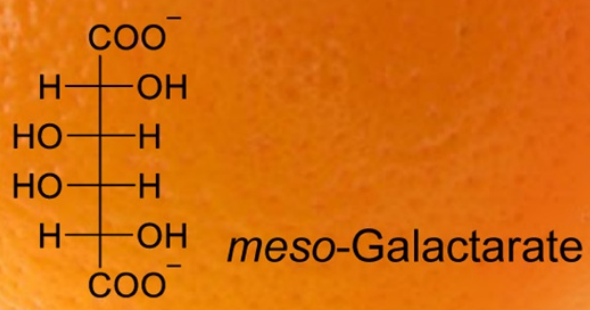

Identification and characterization of a galacturonic acid transporter from Neurospora crassa and its application for Saccharomyces cerevisiae fermentation processes

Benz et al. 


\title{
Identification and characterization of a galacturonic acid transporter from Neurospora crassa and its application for Saccharomyces cerevisiae fermentation processes
}

\author{
J Philipp Benz ${ }^{1 *}$, Ryan J Protzko ${ }^{1,2}$, Jonas MS Andrich ${ }^{1,5}$, Stefan Bauer ${ }^{1}$, John E Dueber ${ }^{1,3}$ and Chris R Somerville ${ }^{1,4}$
}

\begin{abstract}
Background: Pectin-rich agricultural wastes potentially represent favorable feedstocks for the sustainable production of alternative energy and bio-products. Their efficient utilization requires the conversion of all major constituent sugars. The current inability of the popular fermentation host Saccharomyces cerevisiae to metabolize the major pectic monosaccharide D-galacturonic acid (D-GalA) significantly hampers these efforts. While it has been reasoned that the optimization of cellular D-GalA uptake will be critical for the engineering of D-GalA utilization in yeast, no dedicated eukaryotic transport protein has been biochemically described. Here we report for the first time such a eukaryotic D-GalA transporter and characterize its functionality in S. cerevisiae.
\end{abstract}

Results: We identified and characterized the D-GalA transporter GAT-1 out of a group of candidate genes obtained from co-expression analysis in N. crassa. The N. crassa $\Delta$ gat- 1 deletion strain is substantially affected in growth on pectic substrates, unable to take up D-GalA, and impaired in D-GalA-mediated signaling events. Moreover, expression of a gat-1 construct in yeast conferred the ability for strong high-affinity D-GalA accumulation rates, providing evidence for GAT-1 being a bona fide D-GalA transport protein. By recombinantly co-expressing D-galacturonate reductase or uronate dehydrogenase in yeast we furthermore demonstrated a transporter-dependent conversion of D-GalA towards more reduced (L-galactonate) or oxidized (meso-galactaric acid) downstream products, respectively, over a broad concentration range.

Conclusions: By utilizing the novel D-GalA transporter GAT-1 in S. cerevisiae we successfully generated a transporter-dependent uptake and catalysis system for D-GalA into two products with high potential for utilization as platform chemicals. Our data thereby provide a considerable first step towards a more complete utilization of biomass for biofuel and value-added chemicals production.

Keywords: Pectin, D-galacturonic acid, Neurospora crassa, Sugar transport, Saccharomyces cerevisiae, Metabolic engineering, Bioconversion, Meso-galactaric acid, L-galactonic acid

\footnotetext{
*Correspondence: ph.benz@berkeley.edu

${ }^{1}$ Energy Biosciences Institute, University of California Berkeley, Berkeley, CA, USA

Full list of author information is available at the end of the article
}

\section{Biomed Central}

(C) 2014 Benz et al.; licensee BioMed Central Ltd. This is an Open Access article distributed under the terms of the Creative Commons Attribution License (http://creativecommons.org/licenses/by/2.0), which permits unrestricted use, distribution, and reproduction in any medium, provided the original work is properly credited. The Creative Commons Public Domain Dedication waiver (http://creativecommons.org/publicdomain/zero/1.0/) applies to the data made available in this article, unless otherwise stated. 


\section{Background}

The development of sustainable energy and chemical production methods are among the greatest societal challenges facing our generation. Biofuel and green chemistry efforts utilizing renewable lignocellulosic plant biomass feedstocks contribute substantially towards these goals. However, to maximize economic viability, these processes must implement the complete conversion of all major constituent feedstock sugars. Pectin represents a main plant cell-wall polysaccharide that, unlike cellulose and hemicellulose, is not commonly utilized, especially in Saccharomyces cerevisiae (S. cerevisiae), for biofuel and platform chemical production. Since pectin is most abundant in the primary cell walls of soft and growing tissues, agricultural waste streams from fruits and vegetables are particularly pectin-rich. Crops with a high pectin content of 20 to $>40 \%$ include sugar beet pulp, citrus peels, and apple pomace [1-3], and the use of their respective waste streams for the production of biofuels has been attempted in several studies [4-8]. These residues are naturally largely devoid of lignin, already collected at the processing plant, and partly pretreated during the sugar and juice extraction procedure, warranting their high potential as biofuel feedstocks $[9,10]$. The pectic cell wall fraction can be inexpensively and efficiently hydrolyzed into its component monosaccharides by enzymatic processes, as it is considerably less recalcitrant than other plant polysaccharides, such as cellulose. Despite these advantages, pectin-rich sugar beet pulp and citrus peels are currently disposed of in landfills or dried for use as a low-value cattle feed, an expensive procedure that significantly reduces profit margins. Utilizing these waste streams would augment current biofuel production efforts without creating direct or indirect land-use changes.

Pectin comprises a heterogeneous polysaccharide family with four main structural classes: homogalacturonan (HG), rhamnogalacturonan I (RG-I), and the substituted HGs rhamnogalacturonan II (RG-II) and xylogalacturonan (XG) (for review, see $[11,12]$ ). All pectic structures have $\alpha$-D-galacturonic acid (D-GalA) as a major backbone component linked at the $O-1$ and the $O-4$ positions. This monosaccharide alone can comprise up to $70 \%$ of pectin; the remaining sugars include L-arabinose (L-Ara), D-galactose (D-Gal), L-rhamnose (L-Rha), and D-xylose (D-Xyl).

Engineering an organism to utilize D-GalA promises an attractive route for converting a currently underutilized, relatively abundant sugar into a biofuel or platform chemical. The yeast $S$. cerevisiae is currently the most attractive production host and remains the most popular microorganism for industrial fermentation strategies to produce bioethanol. Its advantages include a high tolerance to growth inhibitors from lignocellulose hydrolysates as well as ethanol, the ability to withstand low $\mathrm{pH}$ conditions that eradicate many bacterial contaminants, fast fermentation kinetics, and the suitability for many rounds of recycling $[13,14]$. Ample engineering efforts have already been undertaken to utilize glucose, xylose, and arabinose. Unfortunately, S. cerevisiae cannot metabolize D-GalA, because it lacks the genes encoding a catabolic pathway [15-17]. When fermenting hydrolysates from pectin-rich feedstocks, this could, therefore, lead to the accumulation of D-GalA in the broth, which was shown to be inhibitory to the fermentation of D-Gal, L-Ara, and D-Xyl [18]. A possible approach to overcome this problem is metabolic engineering. In this case, the genes encoding the necessary enzymes for D-GalA metabolism derived from organisms capable of utilizing this sugar could be heterologously expressed in yeast. Such pathways have been described in bacteria, such as Escherichia coli and Erwinia [19-22], as well as more recently in filamentous fungi, such as Aspergillus niger, Trichoderma reesei (anamorph of Hypocrea jecorina) and Botrytis cinerea [23-28] (also see [29] for further review).

A recent attempt to engineer $S$. cerevisiae strains carrying a bacterial D-GalA catabolic pathway met with considerable challenges in expressing functional enzymes [16,30]. Moreover, even though D-GalA was demonstrated to be able to enter $S$. cerevisiae cells under certain conditions (through an as-yet unidentified, lowaffinity and channel-like pore at acidic $\mathrm{pH}$ close to its $\mathrm{pK}_{\mathrm{a}}$ of about 3.5 [31]), it was reasoned that the optimization of D-GalA transport will be essential for the successful engineering of D-GalA utilization in yeast $[14,16]$. Although prokaryotic D-GalA transport systems are well known [32-35], these are notoriously difficult to express functionally in a eukaryotic host. However, so far no such transport protein has been described in a Eukaryote. Here we report such a eukaryotic D-GalA transporter. The corresponding gene was identified through a transcriptomics analysis of pectin degradation by the model filamentous fungus Neurospora crassa ( $N$. crassa) [36], followed by a genetic analysis and the biochemical characterization of the encoded protein. Moreover, we show the transporter to be functional when heterologously expressed in S. cerevisiae and useful for the conversion of D-GalA to downstream products. Our findings are therefore an important step towards the effective utilization of pectin-rich feedstocks for the production of platform chemicals or biofuels.

\section{Results}

Identification of NCU00988 from N. crassa as a candidate D-galacturonic acid transporter

To identify candidate D-GalA transporters, we took advantage of a recently generated polysaccharide-biased co-expression matrix [37]. In that study, the wholegenome expression pattern of $N$. crassa cultures $4 \mathrm{~h}$ 
after transfer to cellulose, xylan, pectin, orange peel powder, sucrose, or no carbon were hierarchically clustered. Analysis of these transcriptomic data revealed groups of genes that specifically responded to the presence of the three main plant cell-wall polysaccharides cellulose, hemicellulose, and pectin. Of approximately 160 genes encoding major facilitator superfamily (MFS)type transporters in the $N$. crassa genome, only nine were found in the most pectin-specific cluster (10.1). Two of these, the allantoate permease (NCU02653) and the pantothenate transporter gene $l i z 1$, were considered to be less likely candidates due to their homology to non-sugar transporters and were omitted from further analyses. The induction by pectin versus sucrose (as a broadly repressing control condition) for the other seven candidates is depicted in Figure 1A. The three transporter genes with the strongest induction by pectin (>1,000-fold; $\log _{2}>10$ ) were NCU01132, NCU00988, and NCU01231. For these we tested whether they are specifically induced by D-GalA alone. To this end, cultures of $N$. crassa wild-type (WT) pre-grown in sucrose for $16 \mathrm{~h}$ were transferred to either no carbon (de-repression but no induction) or very low concentrations of DGalA $(2 \mu \mathrm{M})$ and incubated for another $4 \mathrm{~h}$. Under these conditions we expected a highly specific response. As shown in Figure 1B, only the NCU00988 gene displayed a significant induction $>2$-fold over the starvation condition without added sugar, whereas NCU01132 and
NCU01231 did not respond at all. Moreover, when grown on Vogel's medium with $1 \%$ citrus peel pectin as the sole carbon source, only the deletion strain for NCU00988 $(\Delta 0988)$ exhibited a marked delay in pectin consumption over time (Figure 1C). Taken together, these data demonstrate that NCU00988 is of major importance for pectin utilization in $N$. crassa, potentially through the transport of the backbone monosaccharide D-GalA. We therefore decided to rename NCU00988 as the most promising candidate gene gat-1, for galacturonic acid transporter-1.

\section{The $N$. crassa deletion strain of NCU00988 ( $\Delta$ gat- 1$)$ is impaired in growth on pectins and unable to take up D-galacturonic acid}

To test whether the loss of GAT-1 leads to a broad or specific phenotype, the corresponding deletion strain ( $\Delta$ gat-1) was grown alongside the $N$. crassa WT on sucrose, xylan, and two pectic carbon sources (polygalacturonic acid (PGA) and citrus peel pectin) and the mycelial dry weight was determined after $1.5,2.0$, or 4.0 days, respectively, depending on the carbon source (Figure 2A). Under these conditions, the amount of accumulated biomass was found to be significantly reduced only on the pectic substrates, corroborating our earlier findings.

We next set out to test whether the growth inhibition on pectin could be directly linked to loss of D-GalA transport activity of the deletion strain. For this, sucrose
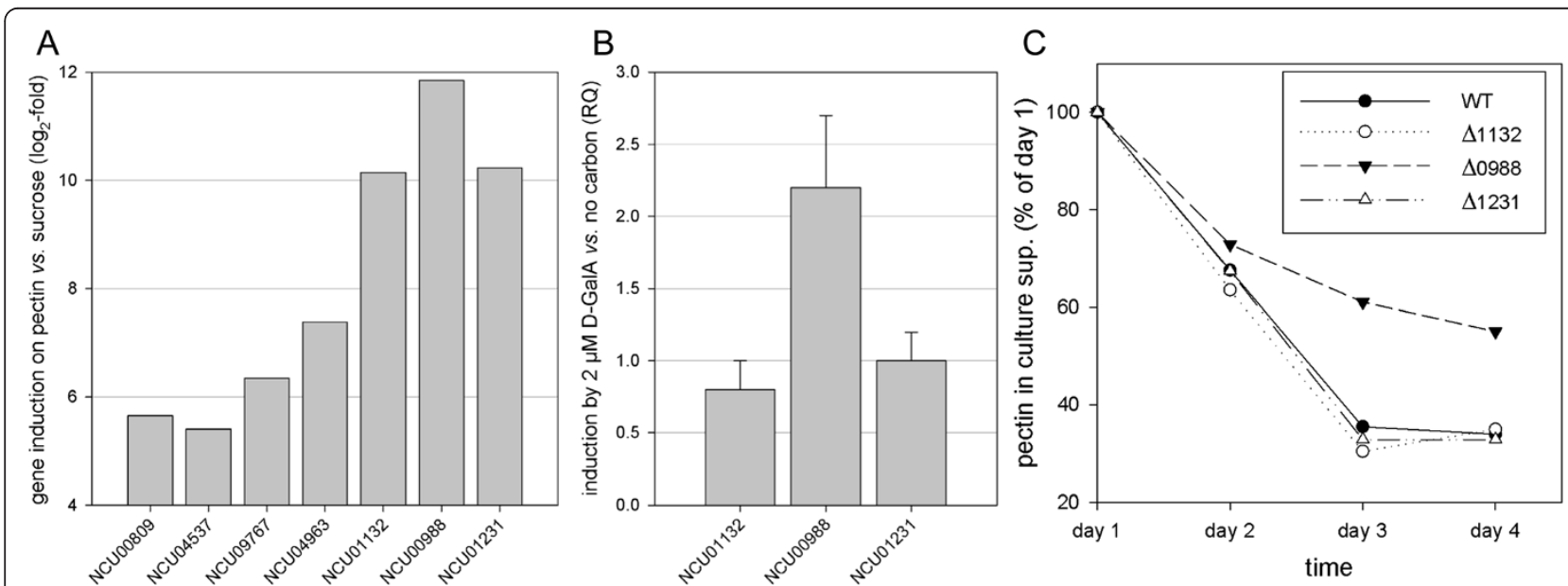

Figure 1 Identification of NCU00988 as a candidate D-galacturonic acid (D-GalA) transporter. (A) Induction of selected transporter genes after transfer for $4 \mathrm{~h}$ to pectin versus sucrose. The $\log _{2}$-fold induction of seven major facilitator superfamily (MFS)-type transporters from the most pectin-specific co-expression cluster as determined by Cuffdiff [37] is shown. Data were calculated from independent triplicate cultures. (B) Relative expression levels of the top three pectin-induced transporter genes in N. crassa as determined by quantitative PCR. Sucrose pre-grown mycelia were transferred to Vogel's medium with $2 \mu \mathrm{M}$ D-GalA or w/o carbon source (no carbon). Samples were taken $4 \mathrm{~h}$ after transfer. Relative transcript quantities $(\mathrm{RQ})$ are depicted where 1 represents the transcription level on medium w/o carbon source. Only NCU00988 showed a response to the presence of D-GalA. Bars represent standard deviations $(n=3)$. (C) Pectin consumption phenotype. The deletion strains of the top three pectin-responsive transporters were grown for 4 days on 1\% pectin and the consumption of pectin followed over time by measuring the remaining substrate concentration in the culture supernatants by the phenol-sulfuric acid method. Data represent the mean of triplicates and are plotted relative to the values at day 1 (as 100\%). Only the deletion strain for NCU00988 (black triangle) displayed a strong delay in pectin consumption. WT, wild-type. 


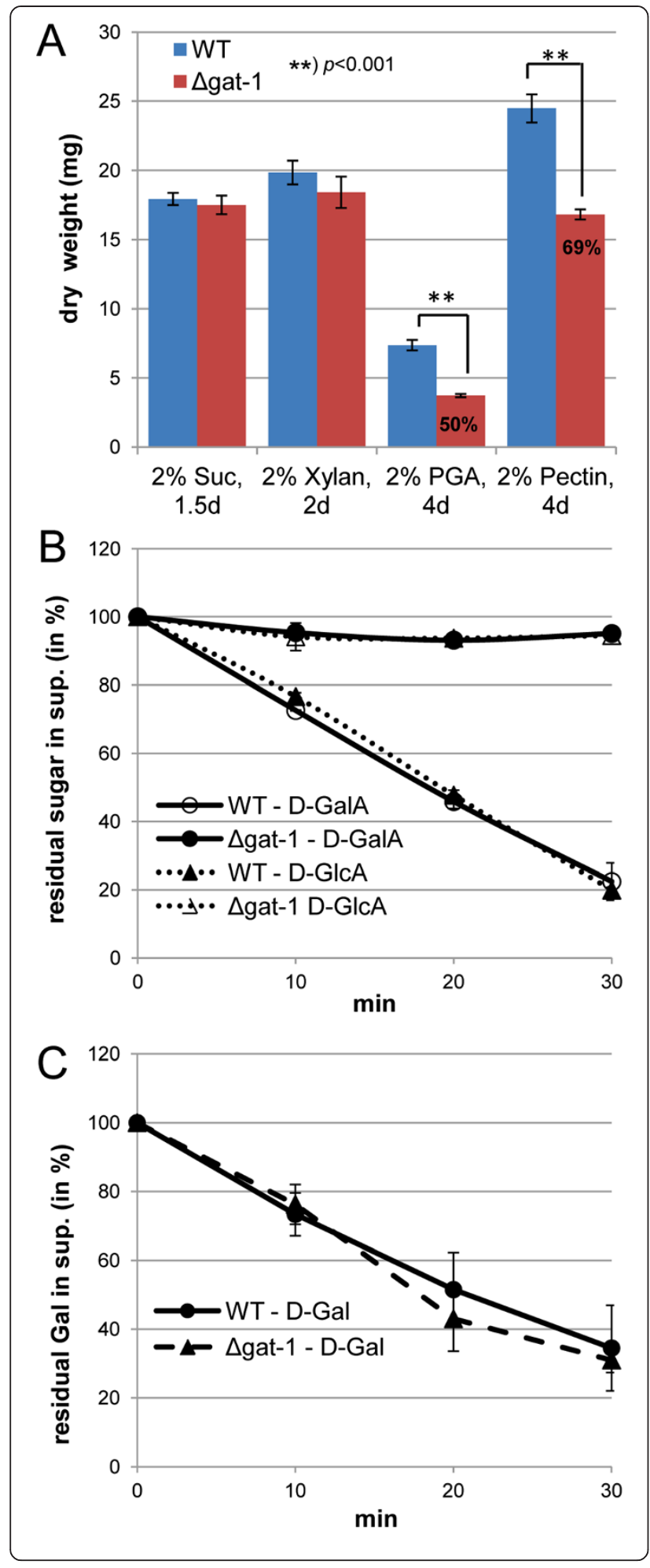

Figure 2 The NCU00988 deletion strain $\Delta$ gat-1 is unable to take up D-galacturonic acid (D-GalA). (A) Carbon source-specific growth phenotype. Conidia of N. crassa wild-type (WT) and the $\triangle$ gat-1 deletion strain were inoculated into 3-mL cultures containing various $2 \%$ carbon sources and incubated at $25^{\circ} \mathrm{C}, 250 \mathrm{rpm}$ in the light. At the indicated times ( 1.5 to 4.0 days) the mycelia were harvested and the dry weight determined. Bars represent standard deviations $(n=5)$. The $\Delta$ gat-1 strain displayed a specific growth reduction on pectic substrates (polygalacturonic acid (PGA) and pectin from citrus peel). The extent of the biomass reduction is indicated (in \% of WT). (B,C) Monosaccharide transport assays. Sucrose pre-grown $N$. crassa WT and $\Delta$ gat-1 mycelia were transferred for $4 \mathrm{~h}$ to $0.5 \%$ pectin to induce the pectinolytic response and subsequently to the reaction solution containing $90 \mu \mathrm{M}$ each of the indicated monosaccharides and Vogel's salts (at pH 5.8). The cultures were incubated in the reaction solution for 30 minutes at $25^{\circ} \mathrm{C}$, $250 \mathrm{rpm}$ in the light. Aliquots of the supernatant were taken at regular intervals and the remaining sugar concentrations analyzed by HPAEC-PAD. Bars represent standard deviations $(n=3)$. $\triangle$ gat- 1 was found to be unable to transport D-GalA as well as D-glucuronic acid (D-GlcA). Suc, sucrose.

pre-grown cultures of WT and $\Delta$ gat-1 were first transferred for an additional $4 \mathrm{~h}$ to Vogel's solution containing $0.5 \%$ pectin as the sole carbon source to induce the pectic response, before being transferred to the reaction solution containing $90 \mu \mathrm{M}$ of either D-Gal, D-GalA, or glucuronic acid (D-GlcA). The residual concentrations of the monosaccharides in the reaction supernatant were measured by high $\mathrm{pH}$-anion exchange-chromatography with pulsed amperometric detection (HPAEC-PAD) and plotted over time in Figures $2 \mathrm{~B}$ and $\mathrm{C}$. Whereas the transport speed for D-Gal was found to be WT-like in the $\Delta g a t-1$ strain (Figure 2C), the transport of both D-GalA and D-GlcA was almost completely abolished (Figure 2B). These findings provide evidence that the physiological function of GAT-1 is indeed the transport of D-GalA (and the minor component D-GlcA) during fungal growth on pectin.

\section{GAT-1 is required for D-GalA-mediated signaling processes in N. crassa}

The near-complete loss of all D-GalA transport activity in the $\Delta$ gat-1 deletion strain suggests that GAT-1 likely represents the only access route for D-GalA in N. crassa, at least under pectin-induced conditions. However, RNAseq data obtained after transfer of mycelia to either sucrose, no carbon, or pectin (see Additional file 1: Figure S1) show that gat-1 is not only strongly induced by pectin but also considerably de-repressed under starvation conditions, and therefore probably part of the carbon scouting machinery [38]. To assess whether the absence of GAT-1 would also have an impact on pectin or, more specifically, D-GalA-mediated signaling events, we analyzed the induction of one of the major polygalacturonase genes (gh28-2) [37] in $\Delta g a t-1$ cultures versus the WT. For this purpose, sucrose pre-grown 
mycelia were transferred to either $0.5 \%$ pectin or $2 \mu \mathrm{M}$ DGalA for $4 \mathrm{~h}$ before harvesting of RNA and measuring the transcript abundance of $g h 28-2$ by quantitative qPCR (Figure 3). The induction of $g h 28-2$ in the $\Delta g a t-1$ strain was found to be strongly reduced on pectin $(<20 \%$ of WT; Figure 3A) and completely undetectable on D-GalA only (Figure 3B). These data corroborate that GAT-1 is required for the uptake of D-GalA and show that it is furthermore an integral part of the fungal pectin signaling pathway.

\section{The transport of D-galacturonic acid and quinic acid are} separate physiological processes

The (generic) annotation of the $N$. crassa genome by the Broad Institute classifies the gat-1 gene (NCU00988) as "MFS quinate transporter", which presumably can be attributed to the fact that GAT-1 and the quinate permease (NCU06026; quinate-Y [39-41]; Broad Institute of Harvard and MIT [http://www.broadinstitute.org/]) indeed share considerable homology (39\% identity, 54\% similarity [42]; Additional file 2: Figure S2). Intriguingly, D-GalA and quinate are also similar in their structure and conformation: quinic acid ( $q a$ ) being a cyclic polyol, with the carboxyl and at least one hydroxyl substituent in identical equatorial conformation and ring position when presented in six-membered ring chair form structures as shown in Additional file 3: Figure S3A. To test whether the two transporters are also functionally redundant, we performed transport assays with $N$. crassa WT mycelia that had been pre-grown in sucrose and were then transferred for $4 \mathrm{~h}$ to Vogel's medium containing both $0.5 \%$ pectin and $100 \mu \mathrm{M} q a$ for the parallel induction of both uptake systems. When the uptake of both substrates was measured over the course of $40 \mathrm{mi}$ nutes, the absence of GAT-1 did not affect qa uptake rates (Additional file 3: Figure S3B), just as the absence of the quinate permease did not negatively affect the uptake of D-GalA (but rather led to a slightly accelerated D-GalA consumption; Additional file 3: Figure S3C). In contrast, the deletion strain for the quinate permease ( $\Delta 6026)$ was almost completely unable to take up $q a$, indicating that it is the only physiologically relevant transporter for this compound. Therefore, despite their homology, our data suggest a distinct, not overlapping role for GAT-1 and the quinate permease in $N$. crassa.

\section{Heterologous expression of GAT-1 in S. cerevisiae confers} the ability to take up D-galacturonic acid with high affinity

As our goal was to find a D-GalA transporter that would be useful for biotechnological applications in yeast, we transformed $S$. cerevisiae with a construct harboring the gat-1 cDNA fused C-terminally to green fluorescent protein (GFP) and driven by the strong phosphoglycerate kinase 1 (PGK1) promoter. Confocal microscopy confirmed correct targeting of the construct into the plasma membrane (Figure 4A). When the yeast cells were subjected to D-GalA uptake assays using $\left({ }^{3} \mathrm{H}\right)-\mathrm{D}-\mathrm{GalA}$ as radio-labeled tracer, we observed substantial D-GalA import over the background of cells transformed with the empty vector (Figure 4B), indicating that GAT-1 was active in the heterologous system. In kinetic experiments with varying D-GalA concentrations, we subsequently determined GAT-1 to be a high-affinity transporter, with a Michaelis constant $\left(\mathrm{K}_{\mathrm{m}}\right)$ value of $1.2+/-0.1 \mu \mathrm{M}$ (mean $+/-\mathrm{SD}$ ) and a maximum velocity $\left(\mathrm{V}_{\max }\right)$ of 12.8 +/- 0.4 nmol minute ${ }^{-1} \mathrm{mg}$ protein $^{-1}$ (Figure $4 \mathrm{C}$ ). The additional observation that D-GalA transport in $N$. crassa is sensitive to the presence of very low concentrations $(30 \mu \mathrm{M})$ of the well-known uncoupling reagent carbonylcyanide $m$-chlorophenylhydrazone (CCCP; Additional file 3: Figure S3D) suggests that GAT-1, which is clearly responsible for the vast majority of D-GalA import under
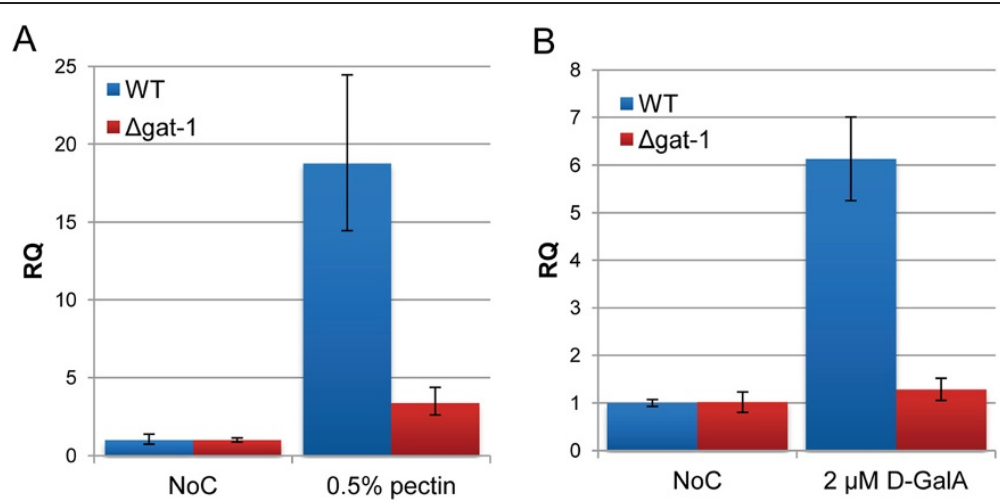

Figure 3 GAT-1 is required for D-galacturonic acid (D-GalA)-mediated induction of pectinases. (A,B) Relative expression levels of the exo-polygalacturonase gene gh28-2 (NCU06961) in N. crassa as determined by quantitative PCR. Sucrose pre-grown WT and $\triangle$ gat-1 mycelia were transferred to medium with $0.5 \%$ citrus peel pectin, $2 \mu \mathrm{M}$ D-GalA or w/o carbon source (NoC). Samples were taken $4 \mathrm{~h}$ after transfer. Relative transcript quantities $(\mathrm{RQ})$ are depicted, where 1 represents the transcription level on NoC. The $\Delta$ gat-1 deletion strain displayed a strong reduction of pectinase induction in both conditions. WT, wild-type. 

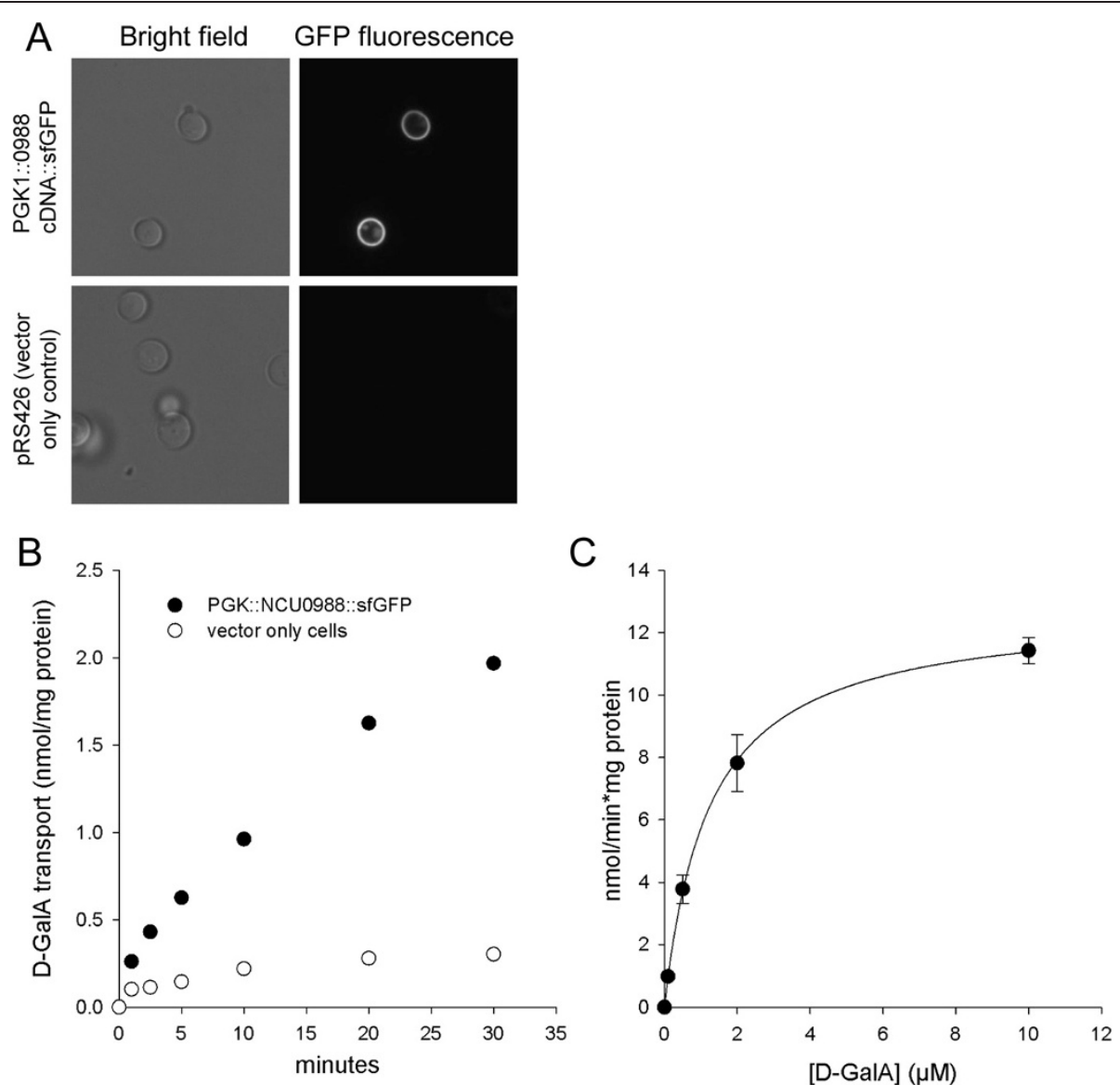

Figure 4 Heterologous expression of gat-1 in yeast confers the ability to uptake D-galacturonic acid (D-GalA) with high affinity. The CDNA of gat-1 was fused to the super folder-green fluorescent protein (sfGFP) under the control of the phosphoglycerate kinase 1 (PGK1) promoter and transformed into yeast (S. cerevisiae strain BY4742). (A) The incorporation of the construct into the plasma membrane was followed by confocal microscopy $(100 \times$ oil; upper panels), whereas no GFP fluorescence could be observed in the vector-only transformed control cells (lower panels). (B) D-GalA transport by the S. cerevisiae strains as described above. Shown is D-GalA transport by yeast with (closed circles) or without (open circles) GAT-1. The initial concentration of D-GalA was $50 \mu \mathrm{M}$. All values are the mean of two measurements. (C) Kinetics of D-GalA transport by GAT-1. The transport rate was determined as a function of D-GalA concentration (ranging from $0.1 \mu \mathrm{M}$ to $10 \mu \mathrm{M}$ ) by yeast strains expressing gat-1-sfGFP and was normalized by total protein concentration.

these conditions, utilizes the proton gradient across the plasma membrane for its function, and therefore likely acts as a $\mathrm{H}^{+} / \mathrm{D}$-GalA symporter.

\section{Engineering of $S$. cerevisiae for the production of L-galactonic acid and meso-galactaric acid from D-galacturonic acid}

To test the utility of GAT-1 in D-GalA bioconversions using $S$. cerevisiae as a production host, we built a strain with gat-1-GFP integrated into the chromosome as well as a strain expressing only GFP from the same chromosomal location, as a control for non-specific uronic sugar transport. These base strains were then transformed to express either Aspergillus niger D-galacturonate reductase (GAAA) or Agrobacterium tumefaciens uronate dehydrogenase (UDH) [26,43], which reduce D-GalA to L-galactonic acid (L-GalOA) or oxidize it to meso- galactaric acid (GalAA), respectively (Figure 5A). We observed rapid uptake in strains expressing the transporter, whereas the D-GalA concentration in the samples of the GFP control strains remained constant during the 3-h assay at high-affinity concentrations $(90 \mu \mathrm{M})$ and $\mathrm{pH} 5.8$ (Figure 5B). The intracellular production of L-GalOA and GalAA, as determined by liquid chromatography coupled to tandem mass-spectrometry (LC-MS/MS) from chloroform:methanol:water-extracted cells, demonstrated production of the respective downstream products in a GAT-1-dependent manner (Figure 5C). Interestingly, whereas GalAA production was enzyme (UDH)dependent, a basal level of incomplete D-GalA bioconversion to L-GalOA was observed without expression of GAAA (strain -/GAT-1). However, expression of the reductase resulted in a higher product yield (GAAA/ GAT-1). 

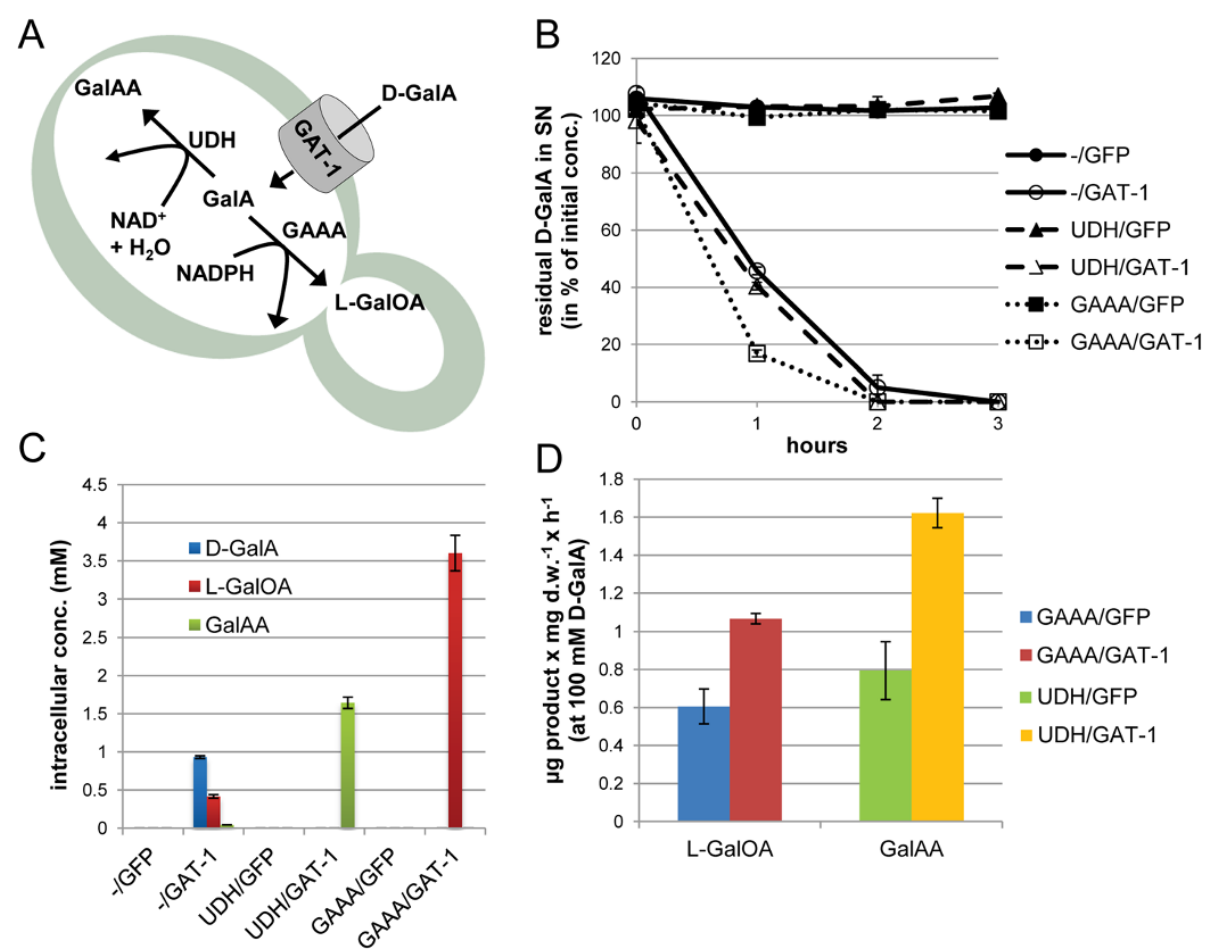

Figure 5 Bioconversion of D-galacturonic acid (D-GalA) to downstream products by genetically engineered yeast strains. (A) D-GalA can be converted to meso-galactaric acid (GalAA) and L-galactonate ( $L-G a \mid O A)$ in S. cerevisiae strains heterologously expressing uronate dehydrogenase $(\mathrm{UDH})$ or D-galacturonic acid reductase (GAAA), respectively, using endogenous cofactors. (B) Bioconversion yeast strains expressing GAT-1 exhibit rapid, high-affinity uptake of D-GalA (at pH 5.8 and an initial D-GalA concentration of 90 MM). (C) Intracellular products were detected by liquid chromatography coupled to tandem mass-spectrometry of chloroform:methanol:water-extracted yeast cells from the 1-h time point samples and their accumulation was found to be transporter-dependent. (D) Even at high D-GalA conditions (100 mM, pH 6.0) co-expression of GAT-1 in GAAA- or UDH-expressing yeast strains increases bioconversion product accumulation by an average 1.8- and 2.1-fold, respectively. $\mathrm{NAD}^{+}$, Nicotinamide adenine dinucleotide; NADPH, Nicotinamide adenine dinucleotide phosphate (reduced); GFP, green fluorescent protein.

Passive uptake of D-GalA by WT yeast has been observed at high concentrations of this substrate (50 to $200 \mathrm{mM}$ ), which is presumably within the concentration range found in hydrolysates from pectin-rich feedstocks. This uptake phenomenon was shown to be largely dependent on the concentration of D-GalA and $\mathrm{pH}$ [31]. The expression of a primary or secondary active transporter could plausibly improve D-GalA transport rates even at high concentrations and/or sustain import as extracellular concentrations decrease. To test whether heterologous expression of the high-affinity transporter GAT-1 will also have beneficial effects in high-D-GalA media conditions we measured L-GalOA and GalAA production in our bioconversion strains at $100 \mathrm{mM}$ D-GalA and $\mathrm{pH}$ 6.0. After $1 \mathrm{~h}$ incubation, we observed an average 1.8-fold improvement in L-GalOA and an average 2.1-fold improvement in GalAA production with expression of GAT-1-GFP over GFP alone (Figure 5D).

\section{Discussion}

In the present study, we identified and characterized the first D-GalA transporter from a eukaryotic organism out of a group of candidate genes derived from a coexpression analysis performed in $N$. crassa. Genetic and biochemical analyses show that the $N$. crassa $\Delta$ gat- 1 deletion strain is substantially affected in growth on pectic substrates and is unable to take up D-GalA from the medium. Moreover, expression of a gat-1 construct in yeast conferred the ability for robust intracellular DGalA accumulation rates, providing evidence for GAT-1 being a bona fide D-GalA transport protein. In view of the fact that the $\Delta g a t-1$ deletion strain is also impaired in D-GalA-mediated signaling events, this furthermore corroborates the notion of an activation system for pectinolytic genes responding to D-GalA ([25] and references therein) and strongly suggests that GAT-1 is an integral part of the D-GalA signaling system in $N$. crassa - either by taking up D-GalA as the signaling molecule (or a precurser thereof) or by acting as a receptor itself.

Several bacterial D-GalA transport systems have already been described, such as ExuT, TogT, TogMNAB and KdgM from E. coli and Erwinia, a bacterial soft-rot causing plant pathogen. KdgM (a porin of the outer membrane [32]), TogMNAB (an $A B C$ superfamily 
member in the inner membrane [33]) and TogT (an MFS-type transporter of the glycoside-pentosidehexuronide (GPH) sub-family also located in the inner membrane [34]) are responsible for D-GalA oligosaccharide transport, whereas ExuT was shown to exclusively transport monomeric D-GalA [35]. Functionally, therefore, GAT-1 seems most similar to ExuT. Indeed, both are high-affinity transporters $\left(\mathrm{K}_{\mathrm{M}}\right.$ of $25 \mu \mathrm{M}$ for ExuT versus $1.2 \mu \mathrm{M}$ for GAT-1), display uptake rates in a very similar range $\left(\mathrm{V}_{\max }\right.$ of $38 \mathrm{nmol} \mathrm{m^{-1 }} \mathrm{mg}^{-1}$ protein $^{-1}$

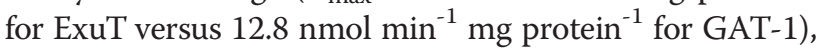
transport D-GlcA in addition to D-GalA and are sensitive to the presence of uncouplers [35]. However, it is noteworthy to mention that the kinetic parameters of GAT-1 were recorded as super-folder (sf)GFP fusion protein heterologously expressed in S. cerevisiae, and that therefore the behavior of the native protein in $N$. crassa might differ from these values. Despite belonging to the same MFS-type superfamily, ExuT (345 aa in $E$. chrysanthemi) and GAT-1 (537 aa in N. crassa) do not share any recognizable protein or sequence similarity and, therefore, have to be considered functional analogs.

Orthologs of GAT-1 (as well as the quinate permease) are found throughout species of Ascomycota and Basidiomycota (see Additional file 2: Figure S2), with the notable exception of Tuber melanosporum, which only seems to have a quinate permease and no additional homolog of GAT-1, and S. cerevisiae, which does not have a clear ortholog of either. Our phylogenetic analysis shows that GAT-1 homologs can be clustered into three clades, each with representatives from both Ascomycota and Basidiomycota (Additional file 2: Figure S2). It furthermore suggests that the GAT-1-containing clade 1 and the quinate permease clade both share a common ancestor and are equally derived from clade 2 and the more distantly related clade 3 . While this manuscript was in proof, Enquist-Newman et al. (2014) discovered another uronic acid transporter (DHT1 from Asteromyces cruciatus) for the uptake of the alginate monomer 4-deoxy-L-erythro-5-hexoseulose uronate (DEHU), which represents a homolog to GAT-1 and the quinic acid permeases and is another good example of the fact that these two transporter families are evolutionarily well related [44].

Considering the number of GAT-1 homologs per genome, Botrytis cinerea seems most diverse, having GAT-1 homologs in all three clades, whereas others, such as Aspergillus nidulans and Trichoderma reesei, have one GAT-1 ortholog (in clade 1) and one or two paralogs in clade 2, respectively. Interestingly, Aspergillus niger only has a clade- 2 homolog to GAT-1. However, since the corresponding gene (An14g04280) has been previously shown to be coregulated with genes involved in pectin degradation and D-GalA catabolism and failed to express in absence of gaaB, the hypothesis of it being a D-GalA transporter has been brought forward [25,45]. Additionally, Zhang et al. (2013) recently reported the An14g04280 homolog in Botrytis cinerea (BcHxt15) to be specifically induced by D-GalA and the corresponding mutant to be impaired in growth on $\mathrm{D}$ GalA, which led them to suggest that BcHXT15 contributes to D-GalA uptake [46]. Even though no representative has been properly biochemically characterized yet, considering these lines of evidence, it seems possible that the clade- 2 homologs are also bona fide D-GalA transport proteins.

Regarding biotechnological applicability, we demonstrated a transporter-dependent conversion of DGalA into two different molecules, L-GalOA and GalAA, by recombinantly expressing D-galacturonate reductase (GAAA) and uronate dehydrogenase (UDH) in yeast, respectively. GalAA is an isomer of glucaric acid, which was identified as a "top valueadded chemical" in a report by the Pacific Northwest National Laboratory and the National Renewable Energy Laboratory for its potential use as a building block for hydroxylated nylons [47-50]. Consequently, GalAA is also likely to be capable of producing desirable hydroxylated nylons. L-GalOA on the other hand is reported to have similar properties to the commodity compound D-gluconic acid and correspondingly holds potential as a chelator in a variety of industries (for example cosmetic, pharmaceutical, food) and can also be used as a chemical precursor to L-ascorbic acid $[45,51,52]$. Production of both of these chemicals was completely GAT-1-dependent at the low D-GalA concentrations typical for highaffinity transport $(90 \mu \mathrm{M})$. Even at high D-GalA concentrations $(100 \mathrm{mM} / 2 \%)$, at which non-specific uronic sugar uptake has been shown to be substantial [31], expression of the transporter increased product accumulation roughly 2 -fold over that observed in control strains at $\mathrm{pH}$ 6.0. Thus, D-GalA utilization can be improved over a broad concentration range in engineered yeast strains expressing a heterologous transporter. Interestingly, whereas production of GalAA was dependent on both GAT-1 and UDH, production of L-GalOA did not require heterologous expression of GAAA. Therefore, a basal level of native D-GalA reductase activity is present in $S$. cerevisiae, though its origin remains unknown.

Utilization of D-GalA for the biosynthesis of biofuels, specifically ethanol, from pectin-rich feedstocks has been a focus of increasing attention in recent years. The isolation and demonstration of activity of this transporter in S. cerevisiae provides an important contribution towards realization of this goal. Although functional expression of 
the requisite pathway enzymes remains an unsolved challenge, an increasing number of alternative orthologous enzymes and pathways are currently being explored [53-55], which will potentially allow this issue to be overcome in the near future.

\section{Conclusions}

In the present manuscript we have for the first time unambiguously identified and biochemically characterized a D-GalA transporter from a eukaryotic origin. GAT-1 was found to be the physiologically relevant D-GalA transporter in $N$. crassa, involved both in the uptake of D-GalA as a metabolite as well as for pectinase signaling events. Moreover, by heterologous expression of GAT-1 in combination with two downstream enzymes, we demonstrate successful transport and catalysis of D-GalA in the fermentation host $S$. cerevisiae, and thereby provide a considerable first step towards a more complete utilization of biomass for biofuel and value-added chemicals production.

\section{Strains, media, and growth conditions}

N. crassa WT (FGSC \#2489) and gene deletion strains used in this study were obtained from the Fungal Genetics Stock Center (FGSC; [www.fgsc.net]). N. crassa was grown on $1 \times$ Vogel's salts [56] with either sucrose (Fisher S3-12; Fisher Scientific, Chicago, IL, USA), or pectin (Sigma P9135; Sigma-Aldrich, St Louis, MO, USA), xylan (beechwood, Sigma X4252), orange peel powder (OPP [37]), or Avicel (PH 101, Fluka 11365) at the indicated concentrations $(\mathrm{w} / \mathrm{v})$ at $25^{\circ} \mathrm{C}$ and $200 \mathrm{rpm}$ with constant light unless stated otherwise.

The yeast strain used in this study was BY4742 (MAT $\alpha$ his $3 \Delta 1$ leu $2 \Delta 0$ lys $2 \Delta 0$ ura3 $\Delta 0$ ) [57]. Growth was usually performed in yeast extract peptone dextrose (YPD) media supplemented with $100 \mathrm{mg} / \mathrm{L}$ adenine hemisulfate and transformed strains were grown in the appropriate complete minimal dropout media, supplemented with $100 \mathrm{mg} / \mathrm{L}$ adenine hemisulfate. Yeast cultures were grown in $250-\mathrm{mL}$ baffled flasks at $30^{\circ} \mathrm{C}$ and $250 \mathrm{rpm}$ for microscopy and uptake experiments.

\section{Plasmids and cloning}

For yeast expression purposes, the $2 \mu$ plasmid pRS426, equipped with the PGK1 promoter and sfGFP as generated and described in [58], was used. The gat-1 cDNA (with optimized Kozak sequence) was inserted between SpeI and ClaI sites using the primers ATACTAGTAAAAATGGG TCTTTCGATAGGAAATAGG and ATATCGATAACATAAACCTCCACATGCTTCG (restriction sites in boldface). For genomic integration, the gat-1 sequence (RefSeq: XM_958805.2) with a C-terminal GFP fusion, or GFP alone, with an upstream TDH3 promoter was flanked by respective 5' and 3' ura3 homology regions using golden- gate cloning. The integration cassette was amplified by PCR and the resulting fragments were used in yeast transformations. The Agrobacterium tumefacians udh open reading frame [GenBank: BK006462.1] and a codon-optimized Aspergillus niger GAAA [GenBank: ABQ53587.1] were cloned into a Cen6/leu2 vector derived from pRS316 [59] with a TDH3 promoter. For used gene sequences, see Table S1 in Additional file 4.

\section{Phenotypic analyses}

Phenotypic analyses of deletion strains were usually performed in 24 deep-well plates in a volume of $3 \mathrm{~mL}$ over the course of 4 days. Dry weight was determined after an overnight incubation of the mycelial mass in aluminum pans in a $105^{\circ} \mathrm{C}$ oven. Pectin consumption over time was followed using the phenol-sulfuric acid assay (PSA) [60]. For this, pectin-grown $N$. crassa cultures were initially cleared by centrifugation at $20,000 \times \mathrm{g}$ for 5 minutes: $2.5 \mu \mathrm{L}$ of the supernatant was diluted into $150 \mu \mathrm{L}$ de-ionized (DI) water and mixed with $150 \mu \mathrm{L}$ of a $5 \%$ phenol solution. Finally, $750 \mu \mathrm{L}$ of concentrated sulfuric acid was quickly added, and the suspension was vortexed for 5 to 10 seconds. After about 7 minutes incubation at room temperature, $200 \mu \mathrm{L}$ of the reactions were analyzed in a plate-reader spectrophotometer (Paradigm; Beckman-Coulter, Brea, CA, USA) at $487 \mathrm{~nm}$.

\section{Phylogenetic analysis}

Protein sequences of GAT-1, NCU06026 (quinate permease), and fungal homologs from representative ascomycete and basidiomycete species were retrieved from the NCBI database. Sequences were aligned and a phylogenetic tree constructed using the program Phylogeny.fr [61] (alignment by MUSCLE - excluding curation by Gblocks, phylogeny calculation by PhyML, and tree rendering by TreeDyn). Gal2p from $S$. cerevisiae was used as the out-group.

\section{Media shift assays for transcriptional studies}

The standard $N$. crassa growth conditions for any experiments involving media switches were as follows: cultures were pre-grown from 9- to 10-day-old conidia for $16 \mathrm{~h}$ in $3 \mathrm{~mL}$ of $1 \times$ Vogel's salts plus $2 \%(\mathrm{w} / \mathrm{v})$ sucrose in 24 deepwell plates. The mycelia were then washed three times in $1 \times$ Vogel's salts without added carbon (NoC) and transferred to $1 \times$ Vogel's salts plus the respective new carbon source (as indicated). The mycelial mass was harvested after an additional $4 \mathrm{~h}$ by quickly blotting dry on Whatman tissues and subsequent flash-freezing in liquid nitrogen to be stored at $-80^{\circ} \mathrm{C}$. Total RNA from frozen samples was isolated using zirconia/silica beads $(0.5 \mathrm{~mm}$ diameter; Biospec, Bartlesville, OK, USA) and a Mini-Beadbeater96 (Biospec) with $1 \mathrm{~mL}$ TRIzol reagent (Invitrogen/Life Technologies, Carlsbad, CA, USA) according to the 
manufacturer's instructions. The total RNA was further digested with TURBO DNA-free (Ambion/Life Technologies) and purified using an RNeasy kit (Qiagen, Valencia, CA, USA). RNA concentration and integrity was checked by Nanodrop and agarose gel electrophoresis.

\section{Quantitative PCR}

Quantitative RT-PCR was performed using the EXPRESS One-Step SYBR GreenER with Premixed ROX kit (Invitrogen/Life Technologies) and the StepOnePlus Real-Time PCR System (Applied Biosystems/Life Technologies). Reactions were performed in triplicate (each from three biological replicates) with a total reaction volume of $10 \mu \mathrm{L}$ including $300 \mathrm{nM}$ each of forward and reverse primers (Additional file 5: Table S2) and 75 ng template RNA. Data analysis was performed by the StepOne Software (Applied Biosystems) using the Relative Quantitation/Comparative CT $(\Delta \Delta \mathrm{CT})$ setting. Data were normalized to the endogenous control actin (NCU04173) with expression on sucrose or no carbon as the reference sample (as indicated).

\section{RNA-Seq}

$N$. crassa cultures were pre-grown from 10-day-old conidia for $16 \mathrm{~h}$ in $100 \mathrm{~mL}$ of $1 \times$ Vogel's salts plus $2 \%$ $(\mathrm{w} / \mathrm{v})$ sucrose using 250-mL shake-flasks. The mycelia were then washed three times in $1 \times$ Vogel's salts without added carbon $(\mathrm{NoC})$ and transferred to $2 \%$ sucrose or $1 \%(\mathrm{w} / \mathrm{v})$ pectin for induction $[37,62]$. After an additional $4 \mathrm{~h}$, the mycelial mass was harvested over a Whatman glass microfiber filter $(\mathrm{GF} / \mathrm{F})$ on a Buchner funnel and subsequently flash-frozen in liquid nitrogen to be stored at $-80^{\circ} \mathrm{C}$. RNA was then prepared as above.

Library preparation for RNA sequencing (RNA-Seq) was performed essentially as described by Benz et al. and Coradetti et al. [37,62]. These were sequenced in SR50 mode on an Illumina HiSeq2000 at the UC Davis Genome Center, and the files analyzed using the Illumina RTA 1.12 software. Mapping of the reads was done against the current version at the time of the $N$. crassa OR74A genome (v10) [39] using Tophat v1.2.0 (http:// tophat.cbcb.umd.edu/) [63]. Transcript abundance was estimated with Cufflinks v0.9.3 in fragments per kilobase of transcript per million mapped reads (FPKMs) using upper quartile normalization and mapping against reference isoforms from the Broad Institute (http://cufflinks. cbcb.umd.edu/) [64-66]. Profiling data are available at the Gene Expression Omnibus (http://www.ncbi.nlm. nih.gov/geo/) [GEO: GSE42692]. Genes with a multiplehypothesis-adjusted $P$-value of $<0.05$ using Cuffdiff were called as significantly differentially expressed between conditions. Independent triplicate cultures were harvested and analyzed for $N$. crassa WT on pectin and sucrose.

\section{Monosaccharide uptake assays}

Transport assays were performed according to [58]. Briefly, $N$. crassa cultures were pre-grown from 9- to 10-day-old conidia for $16 \mathrm{~h}$ in $3 \mathrm{~mL}$ of $1 \times$ Vogel's salts plus $2 \%(\mathrm{w} / \mathrm{v})$ sucrose using 24 deep-well plates. The mycelia were then washed three times in $1 \times$ Vogel's salts without added carbon $(\mathrm{NoC})$ and transferred to $0.5 \%(\mathrm{w} / \mathrm{v})$ pectin for induction. After an additional $4 \mathrm{~h}$, the mycelia were washed again as above and transferred into the uptake buffer $(1 \times$ Vogel's salts plus $90 \mu \mathrm{M}$ monosaccharides or quinic acid, as indicated) for pre-equilibration and to reduce the dilution of the final uptake reaction. Two mycelia of the same genotype were combined into one well to increase the biomass at this stage. In the case of the addition of uncoupler, CCCP (in ethanol at the indicated concentrations; controls with ethanol only) was added at this stage and kept throughout the assay. After 5 minutes, the mycelia were transferred into fresh uptake buffer to start the reaction. Time points of the supernatants were usually taken at 0,5 , and up to 40 minutes. The samples were cleared by centrifugation ( $1 \mathrm{mi}-$ nute at $20,000 \times \mathrm{g}$ ) and $50 \mu \mathrm{L}$ of the supernatant diluted into $450 \mu \mathrm{L}$ of DI water. The monosaccharide concentrations were then quantified by HPAEC-PAD (Thermo Fisher Scientific Inc., Bannockburn, IL, USA): for neutral sugars, a sample size of $25 \mu \mathrm{L}$ was injected onto a Dionex CarboPac PA20 column $(3 \times 30 \mathrm{~mm}$ guard and $3 \times 150 \mathrm{~mm}$ analytical) and eluted at $30^{\circ} \mathrm{C}$ using an isocratic mobile phase of $18 \mathrm{mM} \mathrm{KOH}$ at $0.4 \mathrm{~mL} /$ minute over 11 minutes. For uronic acids, $25 \mu \mathrm{L}$ was injected onto a Dionex CarboPac PA200 column $(3 \times 30 \mathrm{~mm}$ guard and $3 \times 250 \mathrm{~mm}$ analytical) and eluted at $30^{\circ} \mathrm{C}$ using a $50 \mathrm{mM}$ to $170 \mathrm{mM}$ sodium acetate gradient (in $0.1 \mathrm{M} \mathrm{NaOH}$ ) over 8 minutes at $0.4 \mathrm{~mL} /$ minutes.

For mass spectrometry (MS) detection of quinic acid, samples were injected onto a Rezex RFQ Fast Fruit $\mathrm{H}^{+}(8 \%)$ (Phenomenex, Torrance, CA, USA) column (100 $\times$ $7.8 \mathrm{~mm}$ ) and eluted at $55^{\circ} \mathrm{C}$ using an isocratic mobile phase of $0.5 \%$ formic acid at a flow rate of $0.3 \mathrm{~mL} /$ minute. An LTQ XL linear ion trap (Thermo Scientific) was used for MS and MS/MS detection in negative ion mode. The quinic acid precursor ion of $\mathrm{m} / \mathrm{z} 191.2$ was followed (isolation width $\mathrm{m} / \mathrm{z} 2$; CID setting 35; product scan range $\mathrm{m} / \mathrm{z} 50$ to $200)$, and for chromatogram processing the mass transition (MS/MS) of m/z 191.2 to m/z 127.2 was selected.

\section{Yeast bioconversion assays}

Yeast strains were grown as described above and harvested at mid-log phase. Cells were washed twice in $1 / 2 \times$ Vogel's salts ( $\mathrm{pH} 5.8$ ) with $50 \mathrm{mM}$ ethanol added as a nonfermentable carbon source and resuspended in a final volume to yield an optical density $(O D)_{600}$ of 20 . To each experimental condition, $0.5 \mathrm{~mL}$ of concentrated cells were added to 24-well deep-well blocks and $0.5 \mathrm{~mL}$ of $2 \times$ solution was added to yield a final concentration of $1 / 2 \times$ Vogel's 
salts, $90 \mu \mathrm{M}$ D-GalA (Fluka/Sigma-Aldrich 48280-F) and $50 \mathrm{mM}$ ethanol at an $\mathrm{OD}_{600}$ of 10 . Blocks were incubated at $30^{\circ} \mathrm{C}$ with $750 \mathrm{rpm}$ of shaking until the appropriate time point. Samples were cleared by centrifugation and the supernatant was prepared and analyzed as described above. Cell pellets were washed $2 \times$ in cold DI water and freeze-dried in pre-weighed tubes to calculate dry cell weight. For assaying intracellular metabolites, desiccated yeast pellets were subjected to bead-beating (1 minute) in a Mini-Beadbeater-96 (Biospec) with an added $2: 2: 1(\mathrm{v} / \mathrm{v} / \mathrm{v})$ ratio of chloroform, methanol and water. The aqueous phase was collected after centrifugation at $14,000 \mathrm{rpm}$ for 10 minutes and was subsequently concentrated into $60 \mu \mathrm{L}$ of DI water after vacuum centrifugation. The resulting sample was used for MS and MS/ MS detection as described above. The respective precursor ions and mass transitions followed were: D-GalA: $\mathrm{m} / \mathrm{z} 193.1$ to $\mathrm{m} / \mathrm{z}$ 131.1, L-GalOA: $\mathrm{m} / \mathrm{z} 195.1$ to $\mathrm{m} / \mathrm{z}$ 159.1; and GalAA: m/z 209.1 to $\mathrm{m} / \mathrm{z}$ 85.1. For the calculation of intracellular metabolite concentrations, dry cell weight was converted to cell volume using the factor $2.2 \mu \mathrm{L} / \mathrm{mg}$ dry weight $[31,67]$.

High-D-GalA concentration yeast bioconversions were performed in a similar manner as low-concentration uptake assays, but with some modifications. Cells were grown and harvested at mid-log and washed twice in $1 \times \mathrm{PBS}, \mathrm{pH}$ 6.0, with an added $50 \mathrm{mM}$ EtOH. The final cell suspensions in this buffer were adjusted to an $\mathrm{OD}_{600}$ of 20 and $0.5 \mathrm{~mL}$ of cells were added to each condition in a 24 deep-well block in triplicate. To this, $0.5 \mathrm{~mL}$ of solution was added to yield $100 \mathrm{mM}$ D-GalA in $1 \times$ PBS $\left(\mathrm{pH}\right.$ 6.0) with an $\mathrm{OD}_{600}$ of 10 . Blocks were incubated at $30^{\circ} \mathrm{C}$ with $750 \mathrm{rpm}$ of shaking for $1 \mathrm{~h}$. To assay for bioconversion products, a portion of the cell suspension was flash-frozen in liquid nitrogen and added as a 2:2:1 $(\mathrm{v} / \mathrm{v} / \mathrm{v})$ ratio of chloroform, methanol and cell suspension. Washing the cell pellets was avoided to minimize product loss [31]. A duplicate portion of cell suspension of each condition was lyophilized to calculate dry cell weight. Aqueous phase extraction and product detection proceeded as described above.

\section{$\left({ }^{3} \mathrm{H}\right)$-Galacturonic acid transport assays in yeast}

Transport assays were performed using a modified oilstop method [58]. In short, yeast strains expressing gat-1 fused to GFP were grown to an OD $(600 \mathrm{~nm})$ of 4.0 to 5.0 in selective media, washed $3 \times$ with ice cold assay buffer (30 mM MES-NaOH ( $\mathrm{pH} 5.6$ ) and $50 \mathrm{mM}$ ethanol), and resuspended to an OD of 40.0. To start transport reactions, $50 \mu \mathrm{L}$ of cells were added to $50 \mu \mathrm{L}$ of $\left({ }^{3} \mathrm{H}\right.$ )-D-GalA layered over $100 \mu \mathrm{L}$ of silicone oil (Sigma 85419). Reactions were stopped by spinning cells through oil for 1 minute at 17,000 g, tubes were frozen in ethanol/dry ice, and tube-bottoms containing the cell pellets were clipped off into $1 \mathrm{~mL}$ of $0.5 \mathrm{M} \mathrm{NaOH}$. The pellets were solubilized overnight, $5 \mathrm{~mL}$ of Ultima Gold scintillation fluid added, and counts per minute (CPM) determined in a Tri-Carb 2900TR scintillation counter. $\left({ }^{3} \mathrm{H}\right)-\mathrm{D}-\mathrm{GalA}$ was purchased from ViTrax, Inc. (Placentia, CA, USA), and had a specific activity of $25 \mathrm{Ci} / \mathrm{mmol}$ and a purity of $>99 \%$. Kinetic parameters were determined by measuring the linear rate of $\left({ }^{3} \mathrm{H}\right)$-D-GalA uptake over 3 minutes for D-GalA concentrations between 0.1 and $10 \mu \mathrm{M}$. $\mathrm{V}_{\max }$ and $\mathrm{K}_{\mathrm{M}}$ values were determined by fitting a "Ligand binding", "one site saturation" function to a plot of rates versus D-GalA concentrations by nonlinear regression in SigmaPlot ${ }^{\oplus}$. $V_{\max }$ values were normalized by total protein content using a Bradford assay. Kinetic parameters reported in the text are the mean \pm SD from three separate experiments.

\section{Additional files}

Additional file 1: Figure S1. GAT-1 is part of the scouting machinery Transcript abundances in fragments per kilobase of transcript per million mapped reads (FPKM) as determined by RNAseq for the gat-1 gene (NCU00988). Sucrose pre-grown cultures were transferred to either 2\% sucrose, no carbon (NoC), or $1 \%$ pectin for $4 \mathrm{~h}$ before tissue was harvested. Values represent means of three biological replicates (data from [37]). Clearly, gat-1 is subject to carbon catabolite repression in presence of sucrose and is de-repressed under starvation conditions (NoC), thereby assisting in the carbon-scouting of the fungus. The gene is furthermore strongly induced by pectin.

Additional file 2: Figure S2. Maximum likelihood phylogenetic analysis of the GAT-1 transporter. The tree was generated with the help of the phylogeny.fr software workflow [61]. In this case, the alignment was performed by MUSCLE, the phylogeny calculated by PhyML, and the tree rendered by TreeDyn (see Methods). The S. cerevisiae galactose transporter Gal2p was used as out-group. From the Ascomycota: A. nidulans (Aspergillus nidulans, Eurotiomycetes); A.niger (Aspergillus niger, Eurotiomycetes); B.cinerea (Botrytis cinerea, Leotiomycetes); F. graminearum (Fusarium graminearum, Sordariomycetes); M.thermophila (Myceliophthora themophila, Sordariomycetes); M.oryzae (Magnapothe oryzae, Sordariomycetes); N.crassa (Neurospora crassa, Sordariomycetes); P. chrysogenum (Penicillium chrysogenum, Eurotiomycetes); S.cerevisiae (Saccharomyces cerevisiae, Saccharomycotina); S.macrospora (Sordaria macrospora, Sordariomycetes); T.melanosporum (Tuber melanosporum, Pezizomycetes); T.reesei (Trichoderma reesei, Sordariomycetes). From the Basidiomycota: C.gattii (Cryptococcus gattii, Tremellomycetes); L.bicolor (Laccaria bicolor, Agaricomycetes); P.placenta (Postia placenta, Agaricomycetes); U.maydis (Ustilago maydis, Ustilaginomycotina); the arrow indicates the position of GAT-1 in the tree; GAT-1: XP_963898.1; quinate permease: XP_959616.1.

Additional file 3: Figure S3. GAT-1 is not relevant for the uptake of quinic acid and GAT-1-mediated D-galacturonic acid (D-GalA) uptake is inhibited by uncouplers. (A) Cyclic and chair projections of D-GalA and D-quinic acid for comparison. (B-D) Monosaccharide transport assays. Sucrose pre-grown N. crassa mycelia (wild-type (WT) only in D; WT, $\Delta$ gat -1 and $\Delta 6026$ (quinate permease) in $B, C$ ) were transferred for $4 \mathrm{~h}$ to $0.5 \%$ pectin (D) or $0.5 \%$ pectin $+100 \mu \mathrm{M}$ quinic acid $(q a)(B, C)$ to induce the respective response and subsequently to the reaction solution containing $90 \mu \mathrm{M}$ each of GalA (D) or GalA + qa (B,C) and Vogel's salts. The cultures were incubated in the reaction solution for 40 minutes at $25^{\circ} \mathrm{C}, 250 \mathrm{rpm}$ in the light. Aliquots of the supernatant were taken at regular intervals and the remaining sugar/cyclitol concentrations analyzed by High $\mathrm{pH}$ anion-exchange chromatography with pulsed amperometric detection or Linear lon Trap mass spectrometry (LTQ-MS), respectively. Bars represent standard deviations $(n=3)$. 
Additional file 4: Table S1. Codon optimized sequences.

Additional file 5: Table S2. Primers used in quantitative RT-PCR experiments.

\section{Abbreviations}

CCCP: carbonylcyanide m-chlorophenylhydrazone; D-Gal: D-galactose D-GalA: D-galacturonic acid; GalAA: meso-galactaric acid (mucic acid); D-GlcA: D-glucuronic acid; Dl: de-ionized; D-Xyl: D-xylose; GAAA: D-galacturonate reductase; GFP: green fluorescent protein; HG: homogalacturonan; HPAEC-PAD: high pH anion-exchange chromatography with pulsed amperometric detection; L-Ara: L-arabinose; LC-MS/MS: liquid chromatography coupled to tandem mass-spectrometry L-GalOA: L-galactonate; L-Rha: L-rhamnose; MFS: major facilitator superfamily; MS: mass spectrometry; $\mathrm{NAD}^{+}$: Nicotinamide adenine dinucleotide; NADPH: Nicotinamide adenine dinucleotide phosphate (reduced); OD: optical density; PBS: phosphate-buffered saline; PGA: poly-galacturonic acid; PGK1: phosphoglycerate kinase 1; qa: quinic acid; qPCR: quantitative PCR; RG-I: rhamnogalacturonan I; RG-II: rhamnogalacturonan II; RQ: relative transcript quantities; RT-PCR: reverse transcriptase PCR; sfGFP: super-folder green fluorescent protein; suc: sucrose; UDH: uronate dehydrogenase; WT: wild-type; XG: xylogalacturonan; YPD: yeast extract peptone dextrose.

\section{Competing interests}

The authors declare that they have no competing interests.

\section{Authors' contributions}

JPB initiated and coordinated the study, performed or was involved in all experiments and drafted the manuscript. RJP performed and analyzed the yeast engineering and bioconversion experiments and contributed to the manuscript draft. JMSA was involved in the design and generation of the yeast constructs and performed initial experiments. SB developed the protocols for MS detection of conversion products and participated in data generation. JED and CRS co-coordinated the study and helped in drafting the manuscript. All authors read and approved the final manuscript.

\section{Acknowledgements}

We want to thank Ana B Ibáñez for excellent technical assistance, Jon Galazka, Yuping Lin and Ligia Acosta-Sampson for help with the set-up of the tritiated kinetics experiments, and Shu-Lun Tang for help with the ChemDraw structures. We thank Luke Latimer for helpful advice and for his comments on the manuscript. We furthermore acknowledge the FGSC and the Neurospora Genome Project for continuous support. This work was supported by grants from the Energy Biosciences Institute to CRS and JED. JPB was supported by a Feodor-Lynen fellowship from the HumboldtFoundation (GER).

\section{Author details}

${ }^{1}$ Energy Biosciences Institute, University of California Berkeley, Berkeley, CA, USA. ${ }^{2}$ Department of Molecular and Cell Biology, University of California Berkeley, Berkeley, CA, USA. ${ }^{3}$ Department of Bioengineering, University of California Berkeley, Berkeley, CA, USA. ${ }^{4}$ Department of Plant and Microbial Biology, University of California Berkeley, Berkeley, CA, USA. ${ }^{5}$ present address: Institute of Environmental and Sustainable Chemistry, Technische Universität Braunschweig, Braunschweig, Germany.

Received: 5 November 2013 Accepted: 15 January 2014 Published: 6 February 2014

\section{References}

1. Doran JB, Cripe J, Sutton M, Foster B: Fermentations of pectin-rich biomass with recombinant bacteria to produce fuel ethanol. Appl Biochem Biotechnol 2000, 84-86:141-152.

2. Kennedy M, List D, Lu Y, Foo LY, Newman RH, Sims IM, Bain PJS, Hamilton B, Fenton G: Apple Pomace and Products Derived from Apple Pomace: Uses, Composition and Analysis. In Analysis of Plant Waste Materials. Edited by Linskens HF, Jackson JF. Berlin, Heidelberg: Springer; 1999:75-119.

3. Rivas B, Torrado A, Torre P, Converti A, Dominguez JM: Submerged citric acid fermentation on orange peel autohydrolysate. J Agric Food Chem 2008, 56:2380-2387.
4. Angel Siles Lopez J, Li Q, Thompson IP: Biorefinery of waste orange peel. Crit Rev Biotechnol 2010, 30:63-69.

5. Grohmann K, Baldwin EA, Buslig BS: Production of ethanol from enzymatically hydrolyzed orange peel by the yeast Saccharomyces cerevisiae. Appl Biochem Biotechnol 1994, 45-46:315-327.

6. Hang YD, Lee CY, Woodams EE, Cooley HJ: Production of alcohol from apple pomace. Appl Environ Microbiol 1981, 42:1128-1129.

7. Rorick R, Nahar N, Pryor SW: Ethanol production from sugar beet pulp using escherichia coli KO11 and Saccharomyces cerevisiae. Biol Eng 2011, 3:199-209.

8. Vendruscolo F, Albuquerque PM, Streit F, Esposito E, Ninow JL: Apple pomace: a versatile substrate for biotechnological applications. Crit Rev Biotechnol 2008, 28:1-12.

9. Edwards MC, Doran-Peterson J: Pectin-rich biomass as feedstock for fuel ethanol production. Appl Microbiol Biotechnol 2012, 95:565-575.

10. Xiao C, Anderson CT: Roles of pectin in biomass yield and processing for biofuels. Front Plant Sci 2013, 4:67.

11. Atmodjo MA, Hao Z, Mohnen D: Evolving views of pectin biosynthesis. Annu Rev Plant Biol 2013, 64:747-779.

12. Mohnen D: Pectin structure and biosynthesis. Curr Opin Plant Biol 2008 $11: 266-277$

13. Amorim HV, Lopes ML, de Castro Oliveira JV, Buckeridge MS, Goldman GH: Scientific challenges of bioethanol production in Brazil. Appl Microbiol Biotechnol 2011, 91:1267-1275.

14. van Maris AJ, Abbott DA, Bellissimi E, van den Brink J, Kuyper M, Luttik MA, Wisselink HW, Scheffers WA, van Dijken JP, Pronk JT: Alcoholic fermentation of carbon sources in biomass hydrolysates by Saccharomyces cerevisiae: current status. Antonie Van Leeuwenhoek 2006, 90:391-418.

15. Barnett JA, Payne RW, Yarrow D (Eds): A guide to identifying and classifying yeasts. Cambridge: Cambridge University Press; 1990.

16. Huisjes EH, Luttik MA, Almering MJ, Bisschops MM, Dang DH, Kleerebezem $M$, Siezen $R$, van Maris AJ, Pronk JT: Toward pectin fermentation by Saccharomyces cerevisiae: expression of the first two steps of a bacterial pathway for D-galacturonate metabolism. J Biotechnol 2012, 162:303-310.

17. Nijkamp JF, van den Broek M, Datema E, de Kok S, Bosman L, Luttik MA, Daran-Lapujade P, Vongsangnak W, Nielsen J, Heijne WHM, Klaassen P, Paddon CJ, Platt D, Kotter P, van Ham RC, Reinders MJT, Pronk JT, de Ridder $D$, Daran JM: De novo sequencing, assembly and analysis of the genome of the laboratory strain Saccharomyces cerevisiae CEN.PK113-7D, a model for modern industrial biotechnology. Microb Cell Fact 2012, 11:36.

18. Huisjes EH, de Hulster E, van Dam JC, Pronk JT, van Maris AJ: Galacturonic acid inhibits the growth of Saccharomyces cerevisiae on galactose, xylose, and arabinose. Appl Environ Microbiol 2012, 78:5052-5059.

19. Ashwell A, Wahba AJ, Hickman J: A new pathway of uronic acid metabolism. Biochim Biophys Acta 1958, 30:186-187.

20. Ashwell G: Enzymes of glucuronic and galacturonic acid metabolism in bacteria. Method Enzymol 1962, 5:190-208

21. Hugouvieux-Cotte-Pattat N, Robert-Baudouy J: Hexuronate catabolism in Erwinia chrysanthemi. J Bacteriol 1987, 169:1223-1231.

22. Kilgore WW, Starr MP: Catabolism of galacturonic and glucuronic acids by Erwinia carotovora. J Biol Chem 1959, 234:2227-2235

23. Hilditch S: Identification of the fungal catabolic D-galacturonate pathway. In PhD thesis. University of Helsinki, Faculty of Biosciences, Department of Biological and Environmental Sciences, Division of Biochemistry; 2010.

24. Hilditch S, Berghall S, Kalkkinen N, Penttila M, Richard P: The missing link in the fungal D-galacturonate pathway: identification of the L-threo-3-deoxy-hexulosonate aldolase. J Biol Chem 2007, 282:26195-26201.

25. Martens-Uzunova E: Assessment of the Pectinolytic Network of Aspergillus niger by Functional Genomics - Insights from the Transcriptome. In PhD thesis. Wageningen University, Laboratory of Microbiology; 2008

26. Martens-Uzunova ES, Schaap PJ: An evolutionary conserved d-galacturonic acid metabolic pathway operates across filamentous fungi capable of pectin degradation. Fungal Genet Biol 2008, 45:1449-1457.

27. Zhang L: Pectin degradation by Botrytis cinerea: recognition of endo-polygalacturonases by an Arabidopsis receptor and utilization of D-galacturonic acid. In PhD thesis. Wageningen University, Laboratory of Phytopathology; 2013

28. Zhang $L$, Thiewes $H$, van Kan JA: The D-galacturonic acid catabolic pathway in Botrytis cinerea. Fungal Genet Biol 2011, 48:990-997.

29. Richard P, Hilditch S: D-galacturonic acid catabolism in microorganisms and its biotechnological relevance. Appl Microbiol Biotechnol 2009, 82:597-604 
30. Huisjes E: Towards fermentation of galacturonic acid-containing feedstocks with Saccharomyces cerevisiae. In PhD thesis. Delft University of Technology, Industrial Microbiology Section, Department of Biotechnology; 2013.

31. Souffriau B, den Abt T, Thevelein JM: Evidence for rapid uptake of D-galacturonic acid in the yeast Saccharomyces cerevisiae by a channel-type transport system. FEBS Lett 2012, 586:2494-2499.

32. Blot N, Berrier C, Hugouvieux-Cotte-Pattat N, Ghazi A, Condemine G: The oligogalacturonate-specific porin KdgM of Erwinia chrysanthemi belongs to a new porin family. J Biol Chem 2002, 277:7936-7944.

33. Hugouvieux-Cotte-Pattat N, Blot N, Reverchon S: Identification of TogMNAB, an ABC transporter which mediates the uptake of pectic oligomers in Erwinia chrysanthemi 3937. Mol Microbiol 2001 41:1113-1123.

34. Hugouvieux-Cotte-Pattat N, Reverchon S: Two transporters, TogT and TogMNAB, are responsible for oligogalacturonide uptake in Erwinia chrysanthemi 3937. Mol Microbiol 2001, 41:1125-1132.

35. San Francisco MJ, Keenan RW: Uptake of galacturonic acid in Erwinia chrysanthemi EC16. J Bacteriol 1993, 175:4263-4265.

36. Znameroski EA, Glass NL: Using a model filamentous fungus to unrave mechanisms of lignocellulose deconstruction. Biotechnol Biofuels 2013, 6:6

37. Benz JP, Chau BH, Zheng D, Bauer S, Glass NL, Somerville CR: A comparative systems analysis of polysaccharide-elicited responses in Neurospora crassa reveals carbon source-specific cellular adaptations. Mol Microbiol 2014, 91:275-99.

38. Delmas S, Pullan ST, Gaddipati S, Kokolski M, Malla S, Blythe MJ, Ibbett R, Campbell M, Liddell S, Aboobaker A, Tucker GA, Archer DB: Uncovering the genome-wide transcriptional responses of the filamentous fungus Aspergillus niger to lignocellulose using RNA sequencing. Plos Genet 2012, 8:e1002875.

39. Galagan JE, Calvo SE, Borkovich KA, Selker EU, Read ND, Jaffe D, FitzHugh W, Ma LJ, Smirnov S, Purcell S, Rehman B, Elkins T, Engels R, Wang S, Nielsen CB, Butler J, Endrizzi M, Qui D, lanakiev P, Bell-Pedersen D, Nelson MA, Werner-Washburne $M$, Selitrennikoff $C P$, Kinsey JA, Braun EL, Zelter A, Schulte $U$, Kothe GO, Jedd G, Mewes W, et al: The genome sequence of the filamentous fungus Neurospora crassa. Nature 2003, 422:859-868.

40. Tang X, Dong W, Griffith J, Nilsen R, Matthes A, Cheng KB, Reeves J, Schuttler HB, Case ME, Arnold J, Logan DA: Systems biology of the qa gene cluster in Neurospora crassa. Plos One 2011, 6:e20671.

41. Whittington HA, Grant S, Roberts CF, Lamb H, Hawkins AR: Identification and isolation of a putative permease gene in the quinic acid utilization (QUT) gene cluster of Aspergillus nidulans. Curr Genet 1987, 12:135-139.

42. Altschul SF, Gish W, Miller W, Myers EW, Lipman DJ: Basic local alignment search tool. J Mol Biol 1990, 215:403-410.

43. Yoon SH, Moon TS, Iranpour P, Lanza AM, Prather KJ: Cloning and characterization of uronate dehydrogenases from Two pseudomonads and Agrobacterium tumefaciens strain C58. J Bacteriol 2009, 191:1565-1573.

44. Enquist-Newman M, Faust AM, Bravo DD, Santos CN, Raisner RM, Hanel A, Sarvabhowman P, Le C, Regitsky DD, Cooper SR, Peereboom L, Clark A, Martinez Y, Goldsmith J, Cho MY, Donohoue PD, Luo L, Lamberson B, Tamrakar P, Kim EJ, Villari JL, Gill A, Tripathi SA, Karamchedu P, Paredes CJ, Rajgarhia V, Kotlar HK, Bailey RB, Miller DJ, Ohler NL, et al: Efficient ethanol production from brown macroalgae sugars by a synthetic yeast platform. Nature 2014, 505:239-243.

45. Kuivanen J, Mojzita D, Wang Y, Hilditch S, Penttila M, Richard P, Wiebe MG: Engineering filamentous fungi for conversion of D-galacturonic acid to L-galactonic acid. Appl Environ Microbiol 2012, 78:8676-8683.

46. Zhang L, Hua C, Stassen JHM, Chatterjee S, Cornelissen M, van Kan JAL: Genome-wide analysis of pectate-induced gene expression in Botrytis cinerea: identification and functional analysis of putative D-galacturonate transporters. Fungal Genet Biol 2013. doi:10.1016/j.fgb.2013.10.002.

47. Kiely DE, Chen L, Lin TH: Synthetic polyhydroxypolyamides from galactaric, xylaric, D-glucaric, and D-mannaric acids and alkylenediamine monomers - Some comparisons. J Polym Sci Pol Chem 2000, 38:594-603.

48. Mojzita D, Wiebe M, Hilditch S, Boer H, Penttila M, Richard P: Metabolic engineering of fungal strains for conversion of D-galacturonate to meso-galactarate. App/ Environ Microbiol 2010, 76:169-175.

49. Moon TS, Yoon SH, Lanza AM, Roy-Mayhew JD, Prather KLJ: Production of glucaric acid from a synthetic pathway in recombinant Escherichia coli. Appl Environ Microb 2009, 75:589-595.
50. Werpy T, Petersen G, Aden A, Bozell J, Holladay J, White J, Manheim A, Eliot D, Lasure L, Jones S: Top Value Added Chemicals From Biomass. Volume 1 Results of Screening for Potential Candidates From Sugars and Synthesis Gas. Department of Energy Report; 2004:1-76.

51. Csiba M, Cleophax J, Petit S, Gero SD: An expedient and practical 3-step synthesis of vitamin-C from a by-product of the sugar-industry - the L-galactono-1,4-lactone pathway. J Org Chem 1993, 58:7281-7282.

52. Roland JF, Cayle T, Dinwoodie RC, Mehnert DW: Fermentation production of ascorbic acid from L-galactonic substrate. 1983. US Patent 4,595,659.

53. Agius F, Gonzalez-Lamothe R, Caballero JL, Munoz-Blanco J, Botella MA, Valpuesta $\mathrm{V}$ : Engineering increased vitamin $C$ levels in plants by overexpression of a D-galacturonic acid reductase. Nat Biotechnol 2003, 21:177-181.

54. Chang YF, Feingold DS: D-Glucaric acid and galactaric acid catabolism by Agrobacterium tumefaciens. J Bacteriol 1970, 102:85.

55. Hamada S, Seike Y, Tanimori S, Sakamoto T, Kishida M: Characterization of D-galacturonate reductase purified from the psychrophilic yeast species Cryptococcus diffluens. J Biosci Bioeng 2011, 111:518-521.

56. Vogel HJ: A convenient growth medium for Neurospora (medium N). Microbial Genetics Bulletin 1956, 13:42.

57. Brachmann CB, Davies A, Cost GJ, Caputo E, Li J, Hieter P, Boeke JD: Designer deletion strains derived from Saccharomyces cerevisiae S288C: a useful set of strains and plasmids for PCR-mediated gene disruption and other applications. Yeast 1998, 14:115-132.

58. Galazka JM, Tian C, Beeson WT, Martinez B, Glass NL, Cate JH: Cellodextrin transport in yeast for improved biofuel production. Science 2010, 330:84-86.

59. Lee ME, Aswani A, Han AS, Tomlin CJ, Dueber JE: Expression-level optimization of a multi-enzyme pathway in the absence of a highthroughput assay. Nucleic Acids Res 2013, 41:10668-10678.

60. Dubois M, Gilles KA, Hamilton JK, Rebers PA, Smith F: Colorimetric method for determination of sugars and related substances. Anal Chem 1956, 28:350-356.

61. Dereeper A, Guignon V, Blanc G, Audic S, Buffet S, Chevenet F, Dufayard JF, Guindon S, Lefort V, Lescot M, Claverie JM, Gascuel O: Phylogeny.fr: robust phylogenetic analysis for the non-specialist. Nucleic Acids Res 2008 36:W465-W469.

62. Coradetti ST, Craig JP, Xiong Y, Shock T, Tian C, Glass NL: Conserved and essential transcription factors for cellulase gene expression in ascomycete fungi. Proc Natl Acad Sci USA 2012, 109:7397-7402.

63. Langmead B, Trapnell C, Pop M, Salzberg SL: Ultrafast and memoryefficient alignment of short DNA sequences to the human genome. Genome Biol 2009, 10:R25.

64. Roberts A, Pimentel H, Trapnell C, Pachter L: Identification of novel transcripts in annotated genomes using RNA-Seq. Bioinformatics 2011, 27:2325-2329.

65. Roberts A, Trapnell C, Donaghey J, Rinn JL, Pachter L: Improving RNA-Seq expression estimates by correcting for fragment bias. Genome Biol 2011, 12:R22.

66. Trapnell C, Williams BA, Pertea G, Mortazavi A, Kwan G, van Baren MJ, Salzberg SL, Wold BJ, Pachter L: Transcript assembly and quantification by RNA-Seq reveals unannotated transcripts and isoform switching during cell differentiation. Nat Biotechnol 2010, 28:511-515.

67. Sant'Ana GD, Paes LD, Paiva AFV, Fietto LG, Totola AH, Tropia MJM, Silveira-Lemos D, Lucas C, Fietto JLR, Brandao RL, Castro ID: Protective effect of ions against cell death induced by acid stress in Saccharomyces. Fems Yeast Res 2009, 9:701-712.

doi:10.1186/1754-6834-7-20

Cite this article as: Benz et al:: Identification and characterization of a galacturonic acid transporter from Neurospora crassa and its application for Saccharomyces cerevisiae fermentation processes. Biotechnology for Biofuels 2014 7:20. 